\title{
Specificity of Immunoreactions: The Importance of Testing Specificity in Each Method
}

\author{
Andrea Lorincz and Zoltan Nusser \\ Laboratory of Cellular Neurophysiology, Institute of Experimental Medicine, 1083 Budapest, Hungary
}

It is widely accepted that the precise subcellular location of a protein critically affects its functional role. Let us demonstrate this point with two elegant examples. Neurotransmitter receptors, clustered directly opposite to the site of neurotransmitter release, are activated in a transient manner, generating fast postsynaptic responses that are precisely time-locked to the presynaptic action potential. In contrast, if the same receptors are distributed in the extrasynaptic membranes far away from synapses, they will be activated by low concentrations of transmitter present in the extracellular space, producing a tonic conductance that is not precisely time-locked to single presynaptic action potentials, but rather echoes the whole network activity on a much slower time scale (Farrant and Nusser, 2005). Another revealing example is the dendritic versus axonal locations of voltage-gated ion channels. Calcium channels present in the dendrites of pyramidal cells play a role in the integration and plasticity of synaptic inputs, whereas the same channels are essential for neurotransmitter release when concentrated at

\footnotetext{
Received June 3, 2008; revised Aug. 4, 2008; accepted Aug. 5, 2008.

A.L. was supported by a János Bolyai Scholarship of the Hungarian Academy of Sciences. Z.N. was the recipient of a Wellcome Trust Project grant, a European Commission Integrated Project grant (EUSynapse Project; LSHM-CT-2005-019055), and a European Young Investigator Award (www.esf.org/euryi). The financial support from these foundations is greatly acknowledged. We thank Drs. Bruce L. Tempel and Carol A. Robbins for kindly providing the Kv1.2 ${ }^{-1-}$ mouse brain.

Correspondence should be addressed to either Andrea Lorincz or Zoltan Nusser, Laboratory of Cellular Neurophysiology, Institute of Experimental Medicine, Hungarian Academy of Sciences, Szigony Street 43, 1083 Budapest, Hungary. E-mail: lorincz@koki.hu or nusser@koki.hu.

D0I:10.1523/JNEUROSCI.2494-08.2008

Copyright $\odot 2008$ Society for Neuroscience $\quad$ 0270-6474/08/289083-04\$15.00/0
}

presynaptic active zones. These examples clearly demonstrate that the same protein could fulfill very different functional requirements when targeted to different subcellular locations. Thus, the question arises of how to determine the position of a protein at high resolution. In the past quarter of a century, immunohistochemistry using specific antibodies (Abs) became the champion of the available methods. Despite the fact that the principles of immunohistochemical reactions and their executions are very simple, many studies have reached erroneous conclusions because of the misinterpretation of nonspecific staining patterns. In recent years, this problem became the focus of many scientific discussions and the necessity of specificity tests is finally being more widely accepted (Rhodes and Trimmer, 2006). We welcome and are happy to contribute to efforts aiming to reach standards for the verification of immunoreaction specificity. Here we would like to draw attention to frequently encountered misconceptions regarding specificity tests in immunohistochemistry.

It is very important to emphasize that the labeling pattern obtained after an immunohistochemical reaction not only depends on the primary $\mathrm{Ab}$, but the whole experimental procedure affects the outcome, including (1) the presentation of the antigen/tissue, (2) the conditions of the $\mathrm{Ab}$ incubation, and (3) the visualization of the Ab-antigen complexes (see Appendix and Table 1). The main message of our article is that not only the specificity of the primary $\mathrm{Ab}$, but that of the whole method must be verified (Pool and Buijs, 1988).

What is the best specificity test for immunohistochemistry? In theory, one should use brain tissues from two animals, the only difference between them being that one brain does not contain the molecule of interest (against which the primary $A b$ was raised), whereas all the rest of the molecules have identical density and distribution. This is more or less the case in conventional knock-out (KO) animals, from which a single molecule is deleted. Unfortunately, they are not the perfect solution for the following two reasons: (1) depending on the deletion strategy, a truncated part of the protein could be expressed in KO animals; (2) the expression of many other proteins (often highly homologous isoforms, which are the most likely candidates for Ab crossreactivity) could change in conventional KO mice (see below). Thus, if one performs immunoreactions on sections from a control and an ideal KO brain, and if all labeling observed in control tissue disappears in the KO brain, one can conclude that all signals obtained under that given experimental condition were a result of specific Ab-antigen interactions. However, what such a control experiment does not tell us is that the $\mathrm{Ab}$ is specific under all conditions. Conversely, if the labeling pattern observed in control tissue under a certain experimental condition remains identical in the KO brain means that under such experimental conditions the labeling cannot be considered specific, but it does not mean that the $\mathrm{Ab}$ is nonspecific. 
Next, we would like to illustrate the importance of the applied experimental conditions in determining the outcome of immunohistochemical reactions. One of the most revealing examples, demonstrating the impact of antigen presentation, came from the ingenious work of Watanabe et al. (1998). These authors aimed to localize the NR2A subunit in the hippocampal CA3 area. When reactions were performed on conventional aldehydefixed tissue sections, their Ab provided an identical cytoplasmic staining in control and NR2A KO mice [Watanabe et al. (1998), their Fig. 3 B, C], offering the obvious interpretation that the $\mathrm{Ab}$ was not specific. However, when the same aldehyde-fixed tissue sections were treated with pepsin before immunoreactions, the same Ab provided a very different, punctate neuropil staining, which completely disappeared in $\mathrm{KO}$ tissue mice [Watanabe et al. (1998), their Fig. 4A,B], demonstrating that the $\mathrm{Ab}$ is perfectly capable of recognizing the NR2A subunit when the antigen is presented in the appropriate way. The dramatic effect of different antigen presentations on the immunolabeling can also be demonstrated with an anti-Kv1.2 subunit Ab (Fig. 1). Conventional immunofluorescent labeling on $4 \%$ paraformaldehyde-fixed mouse cortical sections resulted in a weak, diffuse neuropil labeling (Fig. 1A). However, when the tissue was treated with pepsin, very intensely labeled axon initial segments (AISs) became apparent (Fig. $1 C)$. The complete disappearance of all signals in Kv1.2 KO mice demonstrated that the labeling of both the neuropil and the AISs was the consequence of specific anti-Kv1.2 Ab-antigen interactions (Fig. $1 B, D)$. The AISs were also strongly immunopositive for Nav1.6 subunit (Fig. $1 C$, inset), which did not change in Kv1.2 KO mice (Fig. 1D, inset). Interestingly, Kv1.2 immunolabeling of the specialized axonal plexus of cerebellar basket cells called pinceau was identical in pepsintreated (Fig. 1G) and nontreated (Fig. 1E) tissue sections (Wang et al., 1994; Veh et al., 1995; Rhodes et al., 1997), showing that the subcellular location (cortical AISs vs cerebellar pinceau) of a protein could affect its detectability with an Ab. Do these results mean that the Kv1.2 subunit at cortical AISs can only be visualized after pepsin treatment? No. As illustrated in Figure $1 I$, an enrichment of gold particles was found on the plasma membranes of a layer 2/3 pyramidal cell AIS when postembedding immunogold reactions were performed on Lowicryl resin-embedded

\section{Table 1. List of typical experimental variables affecting the outcome of an immunofluorescent reaction}

1. Varying the duration of perfusion fixation from 10 to $\geq 25$ min
2. Altering the fixative from aldehyde to methanol, acrolein or acetone
3. Varying the concentration of paraformaldehyde (from 1 to $\geq 2 \%$ ) and glutaraldehyde (from 0 to $\geq 0.05 \%$ )
4. Changing the type of buffer in which the aldehyde is diluted from phosphate to borate, acetate, citrate or cacodylate and
a corresponding change in pH from 6.0 to 8.0
5. Varying the duration of postfixation from 0 to $\geq 30$ min
6. Applying antigen retrieval methods (e.g., microwave irradiation, heat treatment or enzymatic digestion)
7. Using free-floating vibratome sections versus cryostat sections attached to glass histological slides
8. Using freeze-thawing or not
9. The presence or absence of detergents in the blocking, primary and secondary Ab solutions
10. Using different molecules for blocking nonspecific labeling (e.g., normal serum, fish skin gelatin, bovine serum albumin,
fetal calf serum, milk powder, etc.)
11. Changing the primary Ab dilution from 1:50 to $\geq 1: 500$
12. Changing the incubation time from overnight at room temperature to $\geq 2 \mathrm{~d}$ at $4^{\circ} \mathrm{C}$
13. Changing the secondary Ab dilution from $1: 50$ to $\geq 1: 500$

non-pepsin-treated cortical sections using the same anti-Kv1.2 Ab. This result further emphasizes the point that whether an $\mathrm{Ab}$ is capable of recognizing its epitope or not critically depends on the method and also on the subcellular location of the protein. What could be the reason for this subcellular location-dependent accessibility? One possible explanation is that some epitopes are masked by some (associated) proteins in a subcellular compartment-dependent manner. The following experimental results seem to support this explanation. The metabotropic glutamate receptor subtype 2 (mGluR2) is very strongly expressed throughout the axosomatodendritic surface of cerebellar Golgi cells (Ohishi et al., 1994; Tamaru et al., 2001). We also obtained such an axosomatodendritic labeling pattern with a C-terminal antimGluR2 Ab on conventionally prepared sections (Fig. 2A). However, when an $\mathrm{N}$-terminal anti-mGluR2 Ab was used under these conditions, only the somata and the proximal dendrites of Golgi cells were labeled mainly intracellularly, suggesting that this is not a good antimGluR2 Ab (Fig. 2C). However, when the same N-terminal anti-mGluR2 Ab is used on pepsin-treated sections, the axosomatodendritc labeling of Golgi cells is revealed (Fig. 2D). The most parsimonious explanation of these experiments is that the epitope recognized by the N-terminal $\mathrm{Ab}$ is masked in conventionally prepared tissue, but the epitope at the $\mathrm{C}$ terminus is not. After antigen retrieval (e.g., pepsin treatment), the epitope at the $\mathrm{N}$ terminus also becomes accessible, allowing the localization of mGluR2 with the seemingly useless $\mathrm{Ab}$ as well. It is important to point out that even knowing these results, it is impossible to predict whether this $\mathrm{N}$-terminal epitope is masked or not after tissue preparation for postembedding im- munogold or freeze-fracture replicalabeling techniques. Here, we have demonstrated the importance of the applied method in determining the outcome of an immunohistochemical reaction and the significance of testing the specificity of labeling under each experimental condition. If the labeling pattern differs between different experimental conditions, only those results for which the specificity has been verified should be considered.

As mentioned above, the frequently observed upregulation or downregulation of related isoforms of the protein of interest in $\mathrm{KO}$ animals [e.g., the complete lack of plasma membrane $\mathrm{GABA}_{\mathrm{A}}$ receptor $\delta$ subunit in $\alpha 6 \mathrm{KO}$ mice (Jones et al., 1997)] could lead to misinterpretation of the results of specificity tests. For example, if an anti-X Ab recognizes not only protein $\mathrm{X}$, but also one of its isoforms $\mathrm{Y}$, and if the expression of protein $\mathrm{Y}$ is downregulated in protein $\mathrm{X} \mathrm{KO}$ animals, the complete lack of labeling in the KO would lead to an erroneous conclusion that all signals under this reaction condition are attributable to a specific $\mathrm{Ab}$-protein $\mathrm{X}$ interaction.

What is then the best solution for proving the specificity of an immunohistochemical reaction? Despite the abovementioned potential problems with conventional $\mathrm{KO}$ animals, their use for verifying the specificity of immunolabeling will remain the number one choice for years to come. A better choice of method is the use of inducible and spatially restricted (e.g., single cell type-restricted) deletion of the protein of interest, which could minimize the chance of compensatory upregulation or downregulation of other proteins. We hope that such conditional $\mathrm{KO}$ animals will be available for many proteins and become the choice of method in the not too distant future. Meanwhile, another appropriate specific- 

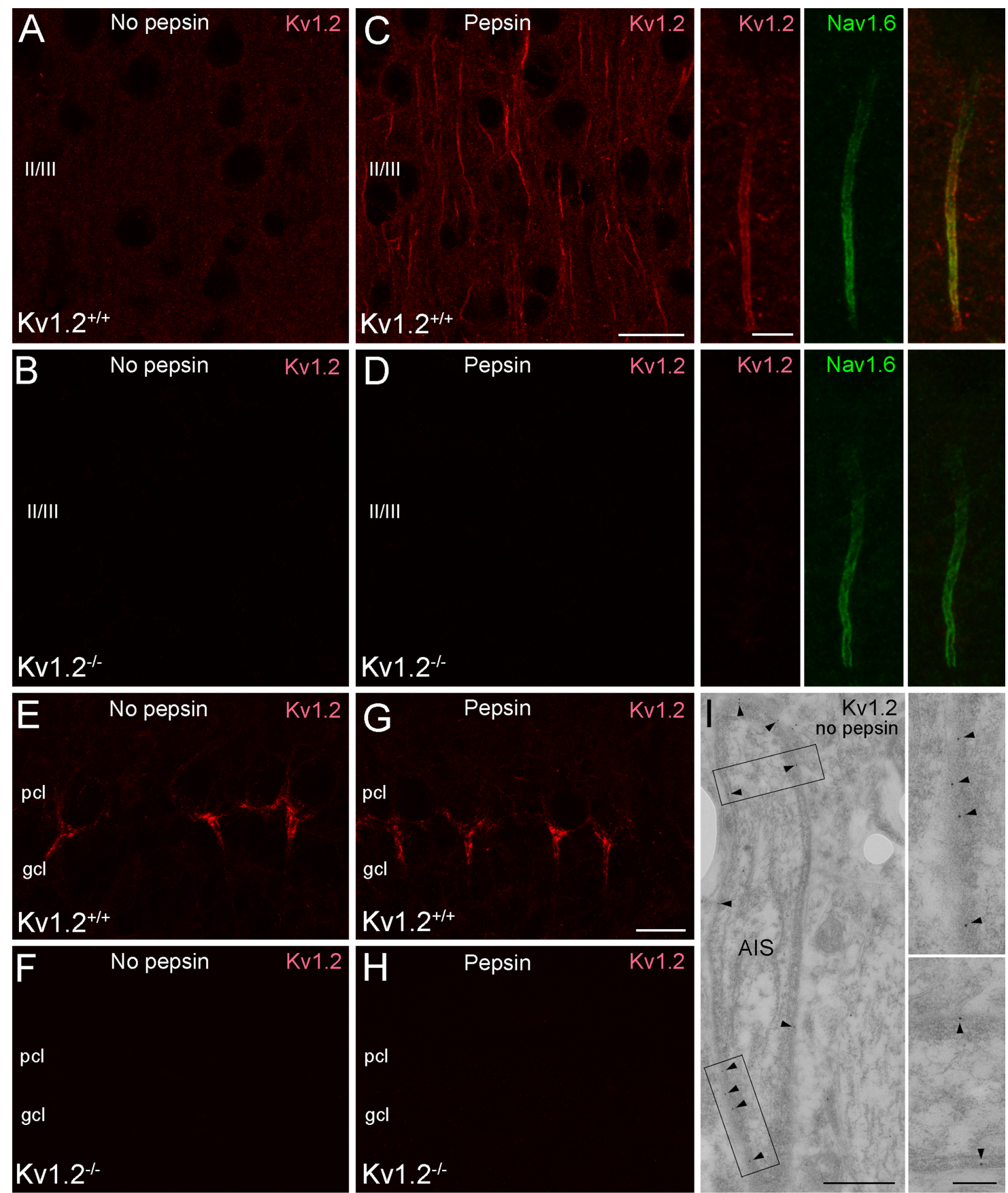

Figure 1. The effect of antigen presentation and subcellular environment on the detectability of the Kv1.2 subunit. $\boldsymbol{A}, \boldsymbol{B}$, Conventional immunofluorescent reaction reveals a weak neuropil labeling for the Kv1.2 subunit in the neocortex of Kv1.2 ${ }^{+/+}$mice $(\boldsymbol{A})$, which completely disappears in Kv1.2 ${ }^{-/-}$mice $(\boldsymbol{B})$. C, In pepsin-treated control neocortical sections, in addition to the neuropil labeling, an intense Kv1.2 subunit immunolabeling appears in AISS. C, Inset, Intense Kv1.2 subunit immunolabeling outlines the plasma membrane of an Nav1.6-subunit-immunopositive AIS. D, In pepsin-treated Kv1.2 $2^{-1-}$ neocortical sections, all immunoreactivity disappeared. $\boldsymbol{E}, \mathbf{G}$, The Kv1.2 subunit immunoreactivity of axonal plexus of cerebellar basket cells (pinceau) is revealed with (G) or without $(\boldsymbol{E})$ pepsin treatment. $\boldsymbol{F}, \boldsymbol{H}$, The labeling disappears in Kv1.2 ${ }^{-I-}$ mice. $\boldsymbol{I}$, An electron micrograph shows postembedding Kv1.2 subunit immunogold reaction in the neocortex. Immunogold particles (arrowhead) associated with the plasma membrane of an AIS could be detected without pepsin treatment. Insets show the boxed areas of the main panel at a higher magnification. Scale bars: (in $\boldsymbol{C}) \boldsymbol{A}-\boldsymbol{D}, 20 \mu \mathrm{m}$; $\boldsymbol{C}, \boldsymbol{D}$, inset, $5 \mu \mathrm{m}$; (in $\boldsymbol{G}) \boldsymbol{E}-\boldsymbol{H}, 20 \mu \mathrm{m} ; \boldsymbol{I}, 500 \mathrm{~nm}$; I, inset, $100 \mathrm{~nm}$.

ity test is using two (or more) antibodies raised against different, nonoverlapping parts of the molecule of interest. If both Abs provide an identical labeling pattern, then it is very likely that all signals are the consequence of a specific Ab-antigen interaction, because the chance of having an additional protein bearing exactly the same two epitope sequences is minuscule. No matter which of these three tests one chooses, the most important point is that the specificity of labeling must be verified under each experimental condition. For example, the complete lack of fluorescent labeling in a $\mathrm{KO}$ tissue section holds no information as to whether gold particles in electron microscopic ultrathin sections or on freeze-fractured replicas are attributable to specific $\mathrm{Ab}$-antigen interactions or not.

In summary, with the help of the above examples we have hoped to highlight the importance of the applied method in determining the outcome of an immunohis- 
tochemical reaction, and also the necessity of performing specificity tests under each experimental condition.

\section{Appendix \\ Summary of distinct experimental conditions with known effects on the outcome of immunoreactions \\ Antigen presentation}

(1) the $\mathrm{pH}$ of the fixative, (2) the concentration of aldehydes in the fixatives, (3) the mode (e.g., immersion or perfusion) of fixation, (4) the duration of fixation, (5) presence or absence of postfixation, (6) application of different antigenretrieval procedures (e.g., pepsin digestion or microwave irradiation), (7) method of freezing, and (8) the type of resin used for embedding the tissue for postembedding reactions, (9) the method of freezing and etching for freeze-fracture replica-labeling reactions.

\section{Conditions of primary $A b$ incubations}

(10) the concentration of the primary Ab, (11) $\mathrm{pH}$ and chemical content (e.g., salt, detergent concentration etc) of the $\mathrm{Ab}$ diluting buffer, (12) the duration and temperature of the incubation.

Visualization of the Ab-antigen complexes (13) direct or indirect preembedding peroxidase reactions with or without amplification, (14) preembedding immunofluorescent reactions, (15) preembedding immunogold reactions (e.g., with large colloidal gold-coupled secondary Abs or with ultrasmall gold-coupled secondary Abs followed by silver enhancement), (16) postembedding immunogold reactions, (17) SDS-etched freeze-fracture replica-labeling reactions.

\section{References}

Farrant M, Nusser Z (2005) Variations on an inhibitory theme: phasic and tonic activation of GABA(A) receptors. Nat Rev Neurosci 6:215-229.

Jones A, Korpi ER, McKernan RM, Pelz R, Nusser Z, Mäkelä R, Mellor JR, Pollard S, Bahn S, Stephenson FA, Randall AD, Sieghart W, Somogyi P, Smith AJ, Wisden W (1997) Ligand-gated ion channel subunit partnerships: $\mathrm{GABA}_{\mathrm{A}}$ receptor
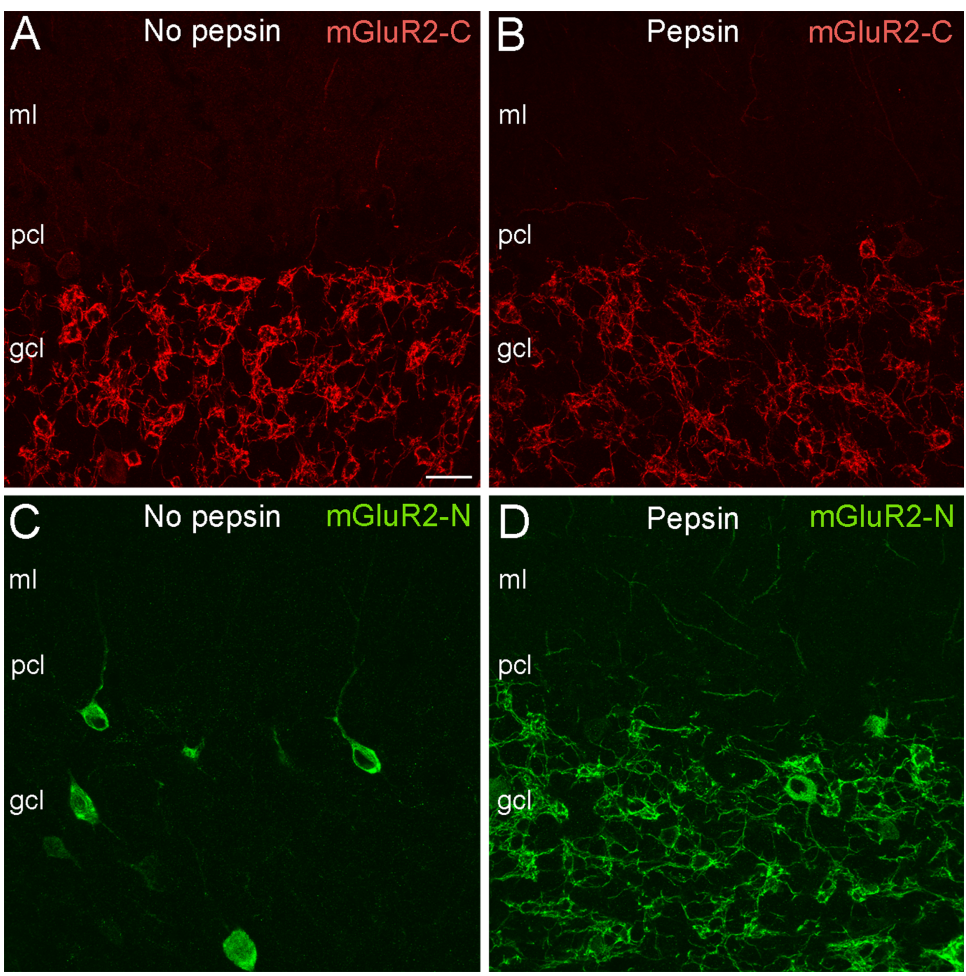

Figure 2. The effect of different antigen presentation depends on the location of the epitope. $A, B, A$ similar axosomatodendritic labeling pattern was detected in cerebellar Golgi cells with $(\boldsymbol{B})$ or without $(\boldsymbol{A})$ pepsin treatment when a $\mathrm{C}$-terminal antimGluR2 antibody (mGluR2-C) was used. $\boldsymbol{C}$, Immunofluorescent reaction with an anti-mGluR2 antibody raised against an $\mathrm{N}$-terminal epitope (mGluR2-N) reveals a somatic and proximal dendritic cytoplasmic labeling of cerebellar Golgi cells. $\boldsymbol{D}$, After pepsin treatment, the mGluR2-N antibody could access the epitopes in all subcellular locations, resulting in the well known axosomatodendritic immunolabeling pattern. All panels are at the same magnification. Scale bar, $20 \mu \mathrm{m}$

$\alpha 6$ subunit gene inactivation inhibits $\delta$ subunit expression. J Neurosci 17:1350-1362.

Ohishi H, Ogawa-Meguro R, Shigemoto R, Kaneko T, Nakanishi S, Mizuno N (1994) Immunohistochemical localization of metabotropic glutamate receptors, mGluR2 and mGluR3, in rat cerebellar cortex. Neuron 13:55-66.

Pool CW, Buijs RM (1988) Antigen identity in immunocytochemistry. In: Molecular neuroanatomy, Vol 3 (Van Leeuwen FW, Buijs RM, Pool CW, and Pach O, eds). Amsterdam: Elsevier.

Rhodes KJ, Trimmer JS (2006) Antibodies as valuable neuroscience research tools versus reagents of mass distraction. J Neurosci 26:8017-8020.

Rhodes KJ, Strassle BW, Monaghan MM, BekeleArcuri Z, Matos MF, Trimmer JS (1997) Association and colocalization of the Kvbetal and Kvbeta2 beta-subunits with Kv1 alphasubunits in mammalian brain $\mathrm{K}+$ channel complexes. J Neurosci 17:8246-8258.
Tamaru Y, Nomura S, Mizuno N, Shigemoto R (2001) Distribution of metabotropic glutamate receptor mGluR3 in the mouse CNS: differential location relative to pre- and postsynaptic sites. Neuroscience 106:481-503.

Veh RW, Lichtinghagen R, Sewing S, Wunder F, Grumbach IM, Pongs O (1995) Immunohistochemical localization of five members of the Kv1 channel subunits: contrasting subcellular locations and neuron-specific co-localizations in rat brain. Eur J Neurosci 7:2189-2205.

Wang H, Kunkel DD, Schwartzkroin PA, Tempel BL (1994) Localization of Kv1.1 and Kv1.2, two K channel proteins, to synaptic terminals, somata, and dendrites in the mouse brain. J Neurosci 14:4588-4599.

Watanabe M, Fukaya M, Sakimura K, Manabe T, Mishina M, Inoue Y (1998) Selective scarcity of NMDA receptor channel subunits in the stratum lucidum (mossy fibre-recipient layer) of the mouse hippocampal CA3 subfield. Eur J Neurosci 10:478-487. 